\title{
EXPLORACIÓN DE LA DIVERSIDAD MORFOLÓGICA DE CHILES REGIONALES EN YAXCABÁ, YUCATÁN, MÉXICO ${ }^{1}$
}

\author{
Luis Latournerie ${ }^{2}$, José Luis Chávez ${ }^{3}$, Manuel Pérez ${ }^{2}$, Carlos F. Hernández ${ }^{2}$, Rodrigo Martínez ${ }^{2}$, \\ Luis M. Arias $^{4}$, Guillermo Castañon ${ }^{2}$
}

\begin{abstract}
RESUMEN
Exploración de la diversidad morfológica de chiles regionales en Yaxcabá, Yucatán, México. El objetivo de este trabajo fue conocer los tipos y usos del chile, los criterios de selección, y obtener muestras de las variantes o poblaciones criollas. El sistema estudiado fue el policultivo maíz, frijol, chile y calabaza (milpa) y se estableció en dos fases: a) una encuesta a 60 familias de la comunidad, a fin de obtener información del cultivo y de los sistemas de cultivo que manejan en la milpa, pach pakal (área apta para hortalizas) y solares. b) una exploración etnobotánica para colectar material genético, y realizar una valoración in situ de los caracteres morfológicos de planta, fruto y flor. En la comunidad, se siembran un total de ocho tipos regionales de chiles, siete pertenecen a Capsicum annuum (yaax ic, xcat'ic, cha'hua, chile dulce, sucurre, pico paloma y maax, este último $C$. annuum var. aviculares) y uno a Capsicum chinense (habanero). El chile yaax ic es el de mayor aceptación en la comunidad. Los agricultores basan la selección de su germoplasma en el tamaño del fruto, sabor, tolerancia a plagas y enfermedades, y usos culinarios. Los chiles regionales se siembran en asociación con otros cultivos tanto en la milpa como en los solares.
\end{abstract}

\begin{abstract}
Morphological diversity exploration of regional peppers in Yaxcaba, Yucatan, Mexico. The main objective of this work was to know the types diversity and uses of the pepper, selection criteria and to get samples samples of the landraces. The studied system was a multi-cropping system with corn, beans, pepper and pumpkin (milpa), established in two stages: a) a survey on 60 land-owners from the community, in order to gather information of the crop and their croppin systems applied in the 'milpa', pach pakal (land useful for gardening) and back-yards. b) An etnobotanical exploration to collect genetic material and to conduct an in situ assessment of the morphological characters of the plant, fruit and flower. Eight regional pepper types are grown in the community, seven belong to the Capsicum annuиm such (yaax ic, xcat'ic, cha'hua, chile dulce, sucurre, pico paloma and maax, the latter $C$. annuum var. aviculare and one $C$. chinense (habanero). The yaax ic pepper is the most widely accepted in the community. The farmers base their germplasm selection on the fruit size, taste, pests and disease tolerance and culinary uses. The regional peppers are planted in association with other crops in the "milpas" as well as in the back-yards.
\end{abstract}

\section{INTRODUCCIÓN}

Actualmente el chile (Capsicum spp.) es uno de los cultivos hortícolas más importantes en el mundo. En 1993, se sembraron más de 1,2 millones de hectáreas con una producción de más de 10,6 millones de toneladas métricas (FAO, 1993). El chile (Capsicum annиum $L$.) junto con el maíz, frijol y la calabaza fueron la base de la alimentación de las culturas Mesoamericanas.
México es el lugar de origen, y centro de distribución y donde se considera que fue domesticado el $C$. апnиит (Hawkes, 1991). El uso de las especies de Capsicum para sazonar la comida, no es una invención reciente, ya que era una práctica común, antes de que Colón llegara a las Antillas. Por lo tanto las diversas manifestaciones culturales y culinarias, del uso de los chiles, formaba parte de la dieta precolombina desde 7500 A.C. (Long, 1988).

\footnotetext{
1 Presentado en la XLVI Reunión Anual PCCMCA, Puerto Rico, 2000.

2 Instituto Tecnológico Agropecuario No.2. Conkal, Yuc., México. E-mail: napoleon @mucuy.itaconkal.edu.mx

3 IPGRI Conservación in situ-Américas-México.

4 CINVESTAV-IPN unidad Mérida. México.
} 
El valor comercial de este cultivo se ha incrementando debido a que se ha diversificado su uso. Además de alimento, condimento, medicina y la industria cosmética, los chiles se usan como plantas ornamentales en jardines (Bosland, 1994). Aunque los chiles no se consideran, como uno de los cultivos de mayor importancia en el mundo, es una de las especies que más se cultiva y tiene importancia económica en algunos países (Mc Leod et al., 1979). Es indispensable un conocimiento amplio de la variabilidad genética, tanto de las variedades silvestres como de las que se cultivan de manera tradicional y de las que se generan a partir del mejoramiento genético. Uno de los principales usos de las variabilidad genética de Capsicum es como fuente de resistencia contra enfermedades. Un ejemplo de la variabilidad genética en $C$. аnnuum es el trabajo de González y Bosland (1991) quienes evaluaron 90 entradas de $C$. baccatum y 35 de $C$. annuum por su resistencia a marchitez por Verticillium.

México es el país del mundo que posee mayor variabilidad genética de Capsicum, pero curiosamente no el productor más importante. En un estudio realizado por la Universidad Autónoma Chapingo y la Universidad Autónoma de Zacatecas, las estadísticas de producción de 1990 ubican a México en el sexto lugar de producción, después de China, España, Turquía, Nigeria e India (Ramírez, 1996). En la Península de Yucatán hay una gran diversidad inter e intraespecífica de tipos de chile que se diferencian por su forma y tamaño, color, sabor y picante. Esta riqueza genética de chiles regionales se debe en gran parte a la diversidad de factores edáficos y climáticos, y a la persistencia de los sistemas tradicionales de cultivo. Los campesinos han manejado sus semillas de chile durante cientos de años en co-evolución con una gran diversidad de patotipos de enfermedades y plagas. Bajo el sistema tradicional los agricultores han adquirido un amplio conocimiento local, producto de la estrecha convivencia que han mantenido, a través de los años, con los ambientes en los que viven y trabajan. Este conocimiento práctico es un recurso valioso que ha permitido al agricultor mantener una gran diversidad genética en sus cultivos. En el ámbito de la conservación de los recursos genéticos los procesos de producción del sistema tradicional como la "milpa" son factores determinantes. Warman (1988) y Bonfil (1990), mencionan que los sistemas agrícolas tradicionales son parte indispensable de la vida de los indígenas mexicanos, al producir los principales alimentos: maíz, frijol, calabaza y chile. Un ejemplo es señalado por Marcus (1982) quien describe que la milpa maya se refiere a campos de maíz cultivado con otros cultivos (frijol, calabaza y chile).
México como otros países, está sufriendo una erosión de germoplasma de chile debido a: a) la pérdida acelerada de los sistemas y tradiciones de la agricultura tradicional, consecuencia de la implementación de proyectos de modernización rural, b) la pérdida de entradas de Capsicum en los bancos de germoplasma y c) el desconocimiento de la diversidad genética que conservan los productores en sus parcelas de cultivo. Todo esto conlleva a un limitado conocimiento sobre la distribución geográfico del género Capsicum en México y más aún sobre la variabilidad de las especies silvestres, cultivadas y los híbridos interespecíficos por el cruzamiento "natural" en los campos de cultivo. Es poco el trabajo que se ha hecho en la protección de las especies silvestres, o domesticadas en sus habitats naturales. Una parte importante en los procesos de conservación in situ es conocer y comprender los factores que influyen en la toma de decisiones de los campesinos para seguir conservando sus recursos genéticos.

Una parte importante de la diversidad genética de chile en México, se encuentra en la Península de Yucatán, donde se conserva como eje de subsistencia la agricultura tradicional (roza-tumba-quema). La carencia de información sistematizada sobre la variabilidad fenotípica y genética de la región Maya, hace necesario iniciar la documentación sobre los tipos de chiles cultivados y silvestres en Yucatán para su aprovechamiento y conservación dado su potencial genético. El presente trabajo se planteó para el área de Yaxcabá, Yucatán con los siguientes objetivos: a) conocer los criterios de selección que utilizan los campesinos para conservar ciclo tras ciclo los diferentes tipos de chile, b) determinar cuáles son los chiles de mayor importancia en la localidad y en dónde prefieren cultivarlo, c) conocer si la diversidad de chiles tiene alguna relación con los principales usos que le dan en la comunidad, y d) colectar diferentes muestras de las variantes o poblaciones criollas.

\section{MATERIALES Y METODOS}

La comunidad de Yaxcabá fue elegido para el presente estudio. Yaxcabá es un municipio localizado en la zona maícera del Estado de Yucatán, México en donde ha perdurado la práctica de la "milpa" tradicional, bajo el sistema de la roza-tumba-quema, como una herencia cultural de los agricultores mayas. En la comunidad, como en muchos lugares de México, se siembra maíz, frijol, calabaza y chile, que se consideran básicos para la alimentación de varias culturas mesoamericanas. La práctica del policultivo, ha creado un sistema agrícola altamente perdurable, ya que el sistema de siembra en 
la milpa, forma parte de la tradición maya. Gracias a esta tradición se ha logrado conservar la biodiversidad de muchas especies nativas en la comunidad.

El municipo de Yaxcabá se ubica entre las coordenadas geográficas de $20^{\circ} 33^{\prime}$ latitud norte y los meridianos $88^{\circ} 50^{\prime}$ de longitud oeste, con una altitud de 30 msnm. Limita al norte con el municipio de Sudzal, al sur con Peto, al este con Sotuta, y al oeste con el municipio de Cuncumul, a una distancia de 108 kilómetros de la ciudad de Mérida, Yucatán. En el área predomina el clima cálido subhúmeda $\left(\mathrm{Aw}_{1}\left(\mathrm{x}^{\prime}\right)\left(\mathrm{i}^{\prime}\right) \mathrm{g}\right)$ con lluvias en el verano, temperatura media anual de $25,5^{\circ} \mathrm{C}$ y una precipitación pluvial media anual de $996,7 \mathrm{~mm}$.

El trabajo consistió de dos etapas. En la primera se llevantó una encuesta en la comunidad de Yaxcabá de abril a mayo de 1999. La comunidad se dividió en cuadrantes de acuerdo a los puntos cardinales, de cada cuadrante se muestreó al azar 15 unidades familiares (60 familias en total) de 540 familias reportadas en la comunidad. El objetivo de la encuesta fue obtener información del cultivo de chile, así como de los sistemas de cultivo de la milpa, el solar o el pach-pakal (superficie de terreno apta para hortalizas, puede estar dentro de la milpa, o alrededor de ésta). Se entrevistaron a las señoras y/o señores de cada familia y por razones culturales la mayoría de las encuestas se hicieron en lengua Maya. Esto permitió obtener tan sólo 13 muestras de chile (ya'axic).

La segunda etapa consistió en una exploración etnobotánica de agosto a diciembre de 1999 en la comunidad objetivo, se colectaron 102 muestras clasificadas preliminarmente dentro de siete morfotipos de $C$. annuum y uno de $C$. chinense (Cuadro 1). Durante la exploración exhaustiva y colección de material genético, se evaluaron in situ varios caracteres morfológicos de fruto, planta y flor de acuerdo con los descriptores para Capsicum (Capsicum spp.) propuestos por el IPGRI, AVRDC y CATIE (1995). Cabe comentar, que en algunas plantas o cultivos de chile, no se logró tomar todos

Cuadro 1. Visión general de los diferentes tipos de chiles cultivados y silvestres en Yaxcabá, Yucatán.

\begin{tabular}{|c|c|c|}
\hline $\begin{array}{r}\text { No. de } \\
\text { colectas }\end{array}$ & Especie & $\begin{array}{c}\text { Tipos por } \\
\text { nombre común }\end{array}$ \\
\hline 72 & C.аппиит & $\begin{array}{l}\text { Xcat'ic } \\
\text { Dulce } \\
\text { Sucurre } \\
\text { Yaax ic } \\
\text { Pico paloma } \\
\text { Cha'huá }\end{array}$ \\
\hline 12 & C. annuum var. aviculare & Maax \\
\hline 18 & C. chinense & Habanero \\
\hline
\end{tabular}

los datos planeados, debido a que estaban fuera del periodo de floración, fructificación, o las plantas estaban en una etapa de senectud, tal vez por efecto de las condiciones adversas del medio ambiente $\mathrm{u}$ otros factores.

Los datos morfológicos fueron colectados en 43 parcelas y solares de 34 productores. Las plantas de estas parcelas, en su mayoría tenían más de un año de edad. Una de las particularidades del trabajo fue el de obtener información tanto del productor como de su esposa, en cada unidad familiar. En la primera visita se entrevistó en forma abierta a la señora con la finalidad de conocer los tipos de chile que ella conserva en el solar, las razones por las que los siguen conservando y los principales usos del fruto o de la planta. Posteriormente, si el agricultor había sembrado chile se visitó la parcela. Las preguntas realizadas al productor fueron similares a las hechas previamente a su esposa, excepto que se le cuestionó acerca del manejo del pachpakal. Para obtener, en lo posible, todos datos morfológicos fue necesario visitar varias veces la misma parcela.

Con la información generada durante la exploración y colección de germoplasma de chile, se hizo un análisis descriptivo de la variabilidad morfológica observada, en cuanto a los tipos establecidos por los productores y su identificación taxonómica, la abundancia en la comunidad, principales criterios usados por los productores para seguir conservando sus fenotipos locales, usos y los sistemas de cultivo donde estos o su esposa siembran las variantes de chile.

\section{RESULTADOS Y DISCUSIÓN}

De acuerdo a los resultados de las encuestas, exploración y colección en la comunidad de Yaxcabá, Yucatán, hay ocho tipos de chiles regionales, siete pertenecen a Capsicum annnum (ya'ax ic, xcat'ic, cha'hua, chile dulce, sucurre, pico paloma y maax o maaxito este último $C$. annuum var. aviculare) y uno a Capsicum chinense (Habanero). De los chiles cultivados, el ya'ax ic es el que siembra el mayor número de productores $38 \%$ (Figura 1). Le sigue el chile habanero (17 $\%$ ) y el chile maax (C. annuum var. aviculare). El ya'ax ic presentó una amplia variabilidad morfológica en la comunidad. Los productores utilizan una serie de nombres locales para designar a las diferentes clases como: yaaxic sac ic (fruto blanco), ya'ax'ic alargado (colmillo) y yaax ic corto entre otros. En México y muchas partes de América, se conoce la ubicación del chile habanero en Yucatán debido a que desde su intro- 


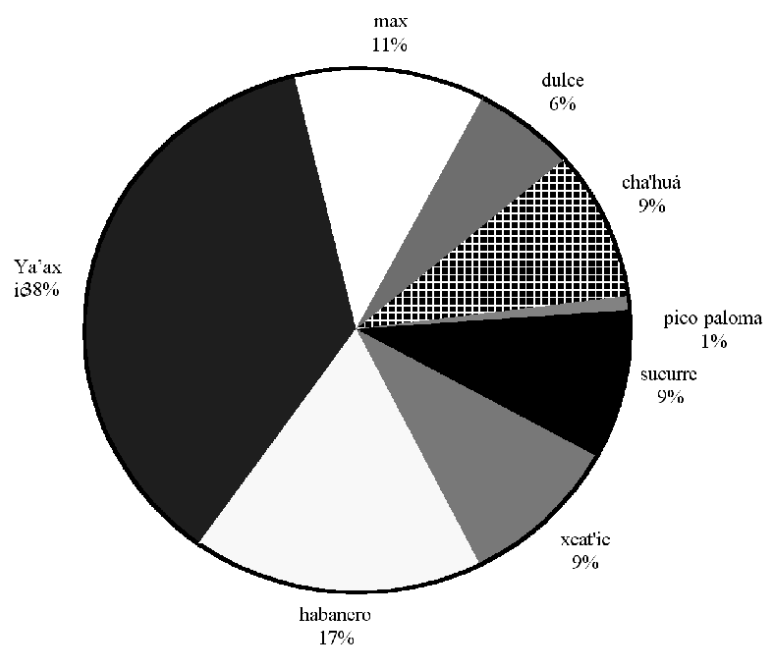

Figura 1. Importancia de los tipos de chile de acuerdo al número de agricultores que lo siembran en Yaxcabá, Yucatán, México. 1999

ducción, encontró un ambiente favorable para su desarrollo, en este caso, la región de Yaxcabá ha encontrado un lugar donde se ha diversificado y las variantes locales son conocidas como habanero (rojo o naranja) y habanero blanco, este último de alta productividad. El maax o maaxito es la especie silvestre local que se encuentra comúnmente en los solares de las casas como plantas individuales y con reducido número de variantes domesticadas. El chile pico paloma es sembrado por el $1 \%$ de los productores. En lo primeros trabajos que reportan Pozo et al. (1991) mencionan reducida variabilidad en contraposición con lo que se encontró en este trabajo. Terán et al. (1998) reportaron que en la comunidad de Socen, Yucatán, se cultivan seis tipos de chile; yaax ic, maax, pico paloma, sucurre, xcat'ic y habanero.

Los criterios que utilizan los productores (as) para seleccionar sus materiales, se centran en una serie de caracteres morfológicos y agronómicos, así como ciertas características culinarias y de procesamiento (Cuadro 2). Así, el chile yaax ic es el preferido de los productores por la forma, color y lo picante de sus frutos, tolerancia a plagas y enfermedades, y su facilidad para la deshidratación. De la última característica sobresalen el cha'hua y maax debido a que los frutos tienen una pared delgada y con la exposición al sol, se obtiene una buena deshidratación. Todo esto ha repercutido en una mayor variabilidad morfológica de yaax ic y en menor grado de cha'hua y maax. De manera local tienen alta demanda en los mercados de la zona. De los chiles cultivados en Yucatán el habanero es el más popular en México de los cultivados en Yucatán por ser el más picante. Por el contrario, el menos picante es el dulce; que, sin embargo, no es el pimento dulce, sweet o bell, sino que es de forma redonda rugosa y similar en tamaño en algunos caso al pimiento.

El yaax ic es el tipo de chile de mayor preferencia por su uso en platillos regionales $(25,6 \%)$. Aunque, a diferencia del chile dulce no se utiliza como platillo principal. La importancia de los chile yaax ic, sucurre y maax tiene que ver también con su uso medicinal y casi todos tienen tienen gran demanda en el mercado tanto en fresco como deshidratado y molido (Cuadro 3).

El yaax ic se siembra en la milpa por la mayoría de los agricultores $(30,39 \%)$. La preferencia para sembrarlo en campo (milpa), además de las características ya mencionadas, al parecer está relacionada con cierta resistencia a la sequía (Cuadro 4); este fenómeno también lo observaron Terán et al. (1998). El solar, la parcela de riego y las macetas, en general, son los sitios donde se encontró al habanero, xcat'ic, maax, dulce y sucurre. El dulce y pico paloma fueron exclusivos de la milpa.

Cuadro 2. Criterios agronómicos y proporción de agricultores que utilizan esos criterios para la selección de los diferentes tipos de chile en Yaxcabá, Yucatán, México.

\begin{tabular}{|c|c|c|c|c|c|c|c|c|}
\hline Criterios & Habanero & Yaax ic & Cha'hua & Xcat'ic & Dulce & Pico paloma & Sucurre & Maax \\
\hline Tamaño fruto & - & 14,50 & 4,60 & 3,80 & 3,0 & 0,76 & 0,76 & 2,30 \\
\hline Forma del fruto & - & 0,76 & - & - & - & - & - & 0,76 \\
\hline Aroma & 3,80 & - & - & - & - & - & - & - \\
\hline Pungencia* & 6,10 & 12,90 & 2,30 & 0,76 & & 0,76 & 4,60 & 6,10 \\
\hline Sabor & 0,76 & - & - & 2,30 & 2,3 & - & - & - \\
\hline Color & 8,40 & 4,60 & - & 1,50 & 1,5 & - & - & - \\
\hline $\begin{array}{l}\text { Tolerancia } \\
\text { patógenos }\end{array}$ & - & 3,80 & - & - & - & - & - & - \\
\hline Deshidratación & - & 2,30 & 0,76 & - & - & - & - & 0,76 \\
\hline Otros & 0,76 & - & - & - & - & - & 1,50 & - \\
\hline Total & 19,82 & 38,86 & 7,66 & 8,36 & 6,8 & 1,52 & 6,86 & 9,92 \\
\hline
\end{tabular}

*Se refiere a lo picante del chile 
Cuadro 3. Porcentaje de preferencias de los productores de los tipos de chile cultivados de acuerdo a su usos culinarios, medicinales y para venta en el mercado.

\begin{tabular}{lcccccccc}
\hline Usos & Habanero & Yaax ic & Cha'hua & Xcat'ic & Dulce & Pico paloma & Sucurre & Maax \\
\hline Platillo principal & - & - & - & - & 2,9 & - & - & - \\
Consumo en fresco & 16,3 & 2,9 & 1,9 & - & - & - & - & - \\
Platillo regional & - & 25,9 & 2,9 & 8,6 & - & - & 0,96 & - \\
Medicinal & - & 1,9 & - & - & - & - & 1,9 & 1,90 \\
Molido & - & 6,7 & 1,9 & - & - & - & 0,96 & 4,80 \\
Venta & - & 2,9 & 2,9 & 0,96 & 2,9 & - & - & 0,96 \\
\hline
\end{tabular}

Por otro lado, se observó que la mayoría de los productores solamente siembran yaax ic, cha'hua y maax (30,39\%, $8,82 \%$ y $7,84 \%$ respectivamente) en la milpa, por lo que la mayoría de los chiles que conservan en la comunidad, se establecen en el solar, de estos, el chile habanero y el sucurre en mayor proporción en macetas de dos a tres plantas (Cuadro 3). Estos resultados están de acuerdo con lo reportado por Aké y Ruenes (1998) quienes mencionan que los solares son un reservorio de conservación in situ.

En importancia, al chile yaax ic, le sigue el chile habanero, el agricultor lo prefiere por el aroma y lo picante, esto lo hace ideal para que tenga una buena aceptación en la comunidad para consumirse en fresco. Este tipo de chile, es sembrado en mayor grado en los solares en macetas y en sistemas de riego (Cuadros 2, 3 y 4). El chile habanero tiene gran aceptación no solo en el estado de Yucatán, sino a nivel internacional. Sin embargo, es muy susceptible a plagas y enfermedades, esto lo hace un cultivo que requiere una fuerte inversión. Los agricultores mayas lo cultivan con tecnologías tradicionales y posiblemente, ésta es una de las razones para que no sea el más importante de la comunidad estudiada.

Cuadro 4. Ubicación de los cultivos de chile dentro de las unidades familiares de 102 productores de Yaxcabá, Yucatán.

\begin{tabular}{lcccc}
\hline Tipo de chile & \multicolumn{4}{c}{ Siembra } \\
\cline { 2 - 5 } & $\begin{array}{c}\text { Milpa } \\
\text { Solar } \\
\mathbf{\%}\end{array}$ & $\begin{array}{c}\text { Riego } \\
\mathbf{\%}\end{array}$ & $\begin{array}{c}\text { Maceta } \\
\mathbf{\%}\end{array}$ \\
\hline Habanero & 0,98 & 3,91 & 4,90 & 6,86 \\
Yaax ic & 30,39 & 5,88 & 0,98 & 1,96 \\
Xcat'ic & - & 0,98 & 4,90 & 2,94 \\
Maax & 7,84 & 0,98 & 0,98 & 0,98 \\
Dulce & - & 1,96 & 1,96 & 1,96 \\
Cha'huá & 8,82 & - & - & - \\
Pico paloma & 0,98 & - & - & - \\
Sucurre & 0,98 & 1,96 & 2,94 & 2,94 \\
Total & $\mathbf{4 9 , 9 9}$ & $\mathbf{1 5 , 6 7}$ & $\mathbf{1 6 , 6 6}$ & $\mathbf{1 7 , 6 4}$ \\
\hline
\end{tabular}

En general, los ocho morfotipos de chile que se siembran en Yaxcabá, presentan características que pre- fieren los productores, desde el tamaño del fruto y sabor hasta el uso que tienen en comidas especiales o bien por su valor agronómico. Los múltiples usos que se les ha dado a los chiles describen la versatilidad de este género. Por lo que en Yucatán los chiles forman parte de la dieta alimenticia, ya que estos son fuentes de vitaminas (Bosland, 1994). Por otro lado la conservación de esta gran diversidad de chiles en la Península de Yucatán esta en manos de los agricultores tradicionales, quienes valoran esta variación basada en las alternativas que le proporcionan como son fuente de alimento, ingresos, y medicinal entre otros debido a las condiciones marginales en los que ellos desarrollan su cultivo.

El pach pakal es la superficie de terreno dentro de la milpa del agricultor seleccionada por ser la porción de terreno apto para sembrar hortalizas. En este se observa un amplio patrón de diferentes cultivos hortícolas de gran importancia para el productor (Figura 2). Entre éstos, se siembra con mayor frecuencia la sandía (12\%), seguido por tomate y chile. El sistema de manejo del pach pakal es en su mayoría de policultivos, en diferentes patrones donde los cultivos comunes son sandía y camote o bien, chile, camote, jícama, tomate. Al respecto Basurto y Castro (1998) mencionan que tanto cul-

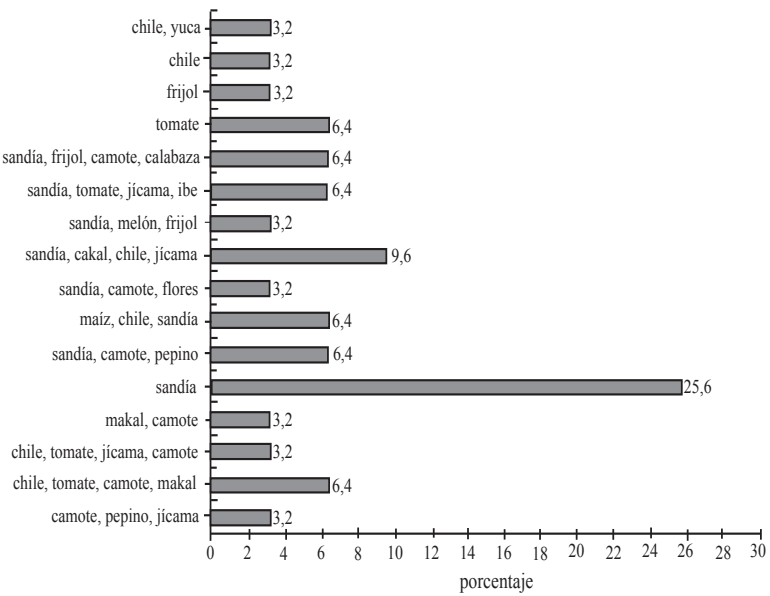

Figura 2. Sistemas de siembras que manejan los agricultores en el pach pakal en Yaxcabá, Yucatán, México. 
tivos domesticados como silvestres que se establecen en el pach pakal representan para el agricultor, un recurso que le proporciona alimento o ingreso monetario antes de la cosecha del cultivo principal.

Los solares o huertos familiares son un sistema agrícola tradicional ampliamente extendido en Yucatán y en diferentes regiones de Mesoamérica. Los huertos familiares son las áreas alrededor de las viviendas que son cultivadas con una gran diversidad de especies de plantas. Tal como se observa en la Figura 3, en los solares se presenta un amplia gama de especies cultivadas desde maíz, chile, tomate, melón, sandía, frijol, yuca, plátano y diversos frutales. Al respecto Medina et al. (1998) mencionan que todo el conocimiento y cúmulo de experiencias que los grupos étnicos tienen sobre el uso y manejo de los recursos naturales, se ve reflejado en el establecimiento de los huertos familiares, para poder satisfacer sus necesidades vitales, y es en estas unidades básicas donde se promueve el cultivo de plantas con diversos usos y para este caso en las unidades familiares de Yaxcabá no fueron la excepción. También Villa y Caballero (1998) mencionan que los solares proporcionan un complemento importante para la nutrición, salud y otras necesidades humanas. Además, pueden representar una fuente de ingresos para los campesinos (Aké y Ruenes, 1998). En este espacio como en el pach pakal la presencia del cultivo de chile es un elemento indiscutible como complementario o fuente principal nutricional de las familias campesinas de Yucatán.

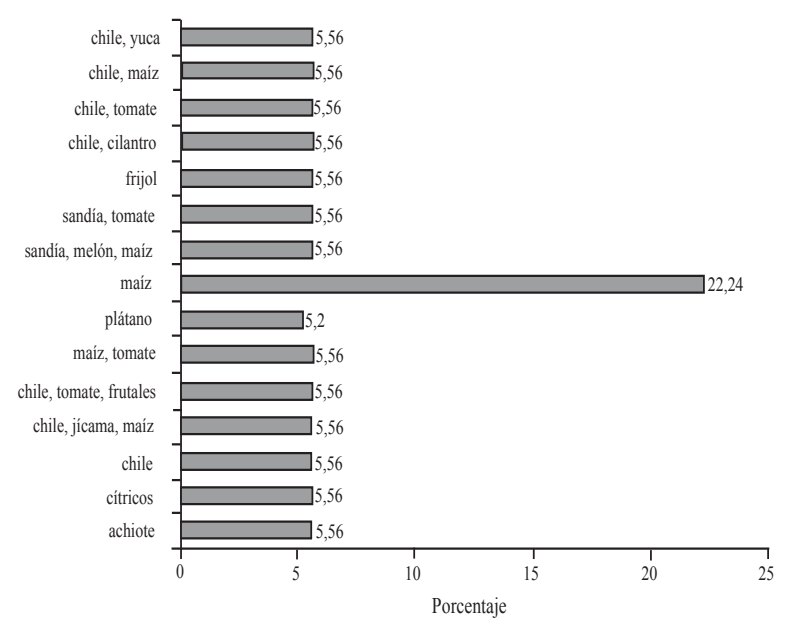

Figura 3. Forma como manejan sus cultivos en los solares las unidades familiares en Yaxcabá, Yucatán, México.

\section{CONCLUSIONES}

En la comunidad de Yaxcabá se colectó un total de 102 poblaciones de chiles criollos, éstas se agruparon en siete tipos de chile que pertencen a $C$. апnиит : chile dulce, yaax ic, xcat'ic, pico paloma, cha'huá, sucurre y maax, $(C$. annumm var. aviculare $)$ y uno a $C$. chinense (habanero). El chile yaax ic es el de mayor importancia en la comunidad por sus amplia variabilidad morfológica, en usos y características agronómicas. El germoplasma de chile es conservado y aprovechado por el campesino maya por las diferentes características que presentan cada uno de éstos, los cuales le permiten satisfacer en parte sus necesidades alimentarias y de ingresos de acuerdo a sus tecnologías tradicionales. Así, los criterios en los que se basan los productores para seleccionar sus materiales son tamaño del fruto, sabor, tolerancia a plagas y enfermedades y usos culinarios, principalmente. Esta diversidad de chiles que manejan los productores en unicultivo o en asociación con otros cultivos (tomate, camote, jícama, etc.) en el pach pakal de la parcela o en el solar son la base principal de una estrategia de conservación in situ de los recursos fitogenéticos tanto de especies silvestre como cultivadas.

\section{LITERATURA CITADA}

AKÉ, A.E.; RUENES, M. 1998. Estudio agroecológico de los huertos familiares de la Península de Yucatán. In: Resúmenes del III Congreso Mexicano de Etnobiología. Oaxaca, México. p. 83.

BASURTO, P.; CASTRO, L. 1998. Cambios en la densidad de anverses útiles (quelites) en milpas y chilares del norte de Puebla. In Resúmenes del III Congreso Mexicano de Etnobiología. Oaxaca, México. p.77.

BOSLAND, P.W. 1994. Chiles: history, cultivation, and uses. In: Charalambous, G. ed. Spices, herbs and edible fungi. Charalambous (ed.) p. 347-366.

BONFIL, G. 1990. México una civilización negada. México. Fondo de la Cultura Económica. 160p.

GONZÁLEZ, M.M.; BOSLAND, P. W. 1991. Germoplasma de capsicum en las Américas. Diversiy 7 (1-2): 57-59.

FAO. 1993. Yearbook production. Vol. 47. Statistics series 117. Rome, Italy. FAO. p.137-138.

HAWKES, J. G. 1991. Centros de diversidad genética vegetal en Latinoamérica. Diversity 7:7-9.

IPGRI (Instituto Internacional de Recursos Fitogenéticos); AVRDC (Centro asiático para el desarrollo y la 
investigación relativos a los vegetales); CATIE (Centro Agronómico Tropical de Investigación y Enseñanza). 1995. Descriptores para Capsicum (Capsicum sp.). IPGRI, AVRDC, CATIE. 48p.

LONG, S. 1988. Capsicum y cultura: la historia del chilli. México. Fondo de la Cultura Económica. 203 p.

MARCUS, J. 1982. The plant world in the sixteenth and seventeenth century lowland Maya. In: Flannery, K. ed. Maya subsistence. Academic press. 310p.

MEDINA M., G.I.; FLORES, M. A.; GARCÍA, G. J. 1998. Etnobiología de los huertos familiares zapotecos en Talea de Castro, Sierra Norte, Oaxaca. In: Resúmenes del III Congreso Mexicano de Etnobiología. Oaxaca, México. p. 79.

MC LEOD, M.J., ESHBAUGH, W. H.; GUTTMAN, S.I. 1979. In: Hawkes, J.; Lester, R.; Skelding, A. eds. The biology and taxonommy of the solanaceae. Linnean society symposium series. No.7. p. 701-713.
POZO, C. O.; MONTES, H.S.; REDONDO, E. 1991. Chile (Capsicum spp.) In: Ortega, R.; Palomino, G.; Castillo, F.; González, V.; Livera, M. eds. Avances en el estudio de los recursos fitogenéticos de México. Sociedad Mexicana de fitogenética, A.C., México, p. 217-238.

RAMÍREZ, J. 1996. El chile. Biodiversidad (México) 2(8): 8-14.

TERÁN, S.; RASMUSSEN, C.H.; MAY, C. O. 1998. Las plantas de la milpa entre los mayas. Yucatán, México. Fundación Tun Ben Kin, A.C. 278 p.

VILLA, K.A.; CABALLERO, N. 1998. Variación florística de los huertos familiares de Guerrero, México. In: Resúmenes del III Congreso Mexicano de Etnobiología. Oaxaca, México. p. 81.

WARMAN, A. 1988. Maíz, la historia de un bastardo. Fondo de la cultura económica. México. 186p. 